Review Article

\title{
Towards Polypharmacokinetics: Pharmacokinetics of Multicomponent Drugs and Herbal Medicines Using a Metabolomics Approach
}

\author{
Ke Lan, ${ }^{1}$ Guoxiang Xie, ${ }^{2}$ and Wei Jia ${ }^{2}$ \\ ${ }^{1}$ Key laboratory of Drug Targeting and Drug Delivery System of the Education Ministry, West China School of Pharmacy, \\ Sichuan University, Chengdu 610041, China \\ ${ }^{2}$ Center for Translational Biomedical Research, University of North Carolina at Greensboro, \\ North Carolina Research Campus, Kannapolis, NC 28081, USA
}

Correspondence should be addressed to Wei Jia; w_jia@uncg.edu

Received 27 December 2012; Accepted 29 January 2013

Academic Editor: Aiping Lu

Copyright (C) 2013 Ke Lan et al. This is an open access article distributed under the Creative Commons Attribution License, which permits unrestricted use, distribution, and reproduction in any medium, provided the original work is properly cited.

Determination of pharmacokinetics (PKs) of multicomponent pharmaceuticals and/or nutraceuticals (polypharmacokinetics, poly-PKs) is difficult due to the vast number of compounds present in natural products, their various concentrations across a wide range, complexity of their interactions, as well as their complex degradation dynamics in vivo. Metabolomics coupled with multivariate statistical tools that focus on the comprehensive analysis of small molecules in biofluids is a viable approach to address the challenges of poly-PK. This paper discusses recent advances in the characterization of poly-PK and the metabolism of multicomponent xenobiotic agents, such as compound drugs, dietary supplements, and herbal medicines, using metabolomics strategy. We propose a research framework that integrates the dynamic concentration profile of bioavailable xenobiotic molecules that result from in vivo absorption and hepatic and gut bacterial metabolism, as well as the human metabolic response profile. This framework will address the bottleneck problem in the pharmacological evaluation of multicomponent pharmaceuticals and nutraceuticals, leading to the direct elucidation of the pharmacological and molecular mechanisms of these compounds.

\section{Introduction}

Multicomponent herbal medicines have gained popularity as complementary interventions against a number of conditions, including metabolic diseases and cancer $[1,2]$. Distinct from the pharmacology of chemical drugs (single compounds), the pharmacology of multi-component agents, including botanical-based nutraceuticals, entails a "network" approach, in which multiple compounds interact in vivo with multiple targets with interdependent activities to achieve an optimal effect $[3,4]$. The vast number of metabolites present in natural products and their wide dynamic range are inextricable obstacles for pharmacological evaluation and nutraceutical/drug development. Furthermore, the coexistence of multiple compounds may lead to metabolic and pharmacokinetic interactions. Due to the complexity of both botanicals and biological samples (e.g., blood, urine, tissues), the analytical approaches to quantitatively measure the time-dependent concentration profiles of bioavailable plant molecules (i.e., poly-PK) are beyond the scope of traditional research. In fact, herbal medicine PK that simultaneously monitors multiple metabolites (i.e., poly-PK) has only been reported in a handful of studies $[5,6]$. As a result, poly-PK has been a long-standing bottleneck in botanical-based medical and nutritional research.

The traditional approach to understand the pharmacology of a multi-component agent is to study the effects of single components on single biological reactions, enzymes, genes, and so forth, and gradually assemble the findings into a whole picture. However, assembling the results obtained from such a reductionist approach to achieve a system understanding of a concerted pharmacological intervention 
has proven impractical [7]. In fact, the results of such attempts failed to accurately capture the complex pharmacokinetic behavior of herbal medicines. Additionally, the PK of a given compound in a multi-component assay may be significantly different from that in a single-compound assay due to drugdrug interactions. With such a complex network involving a large number of variables, it is technically challenging to identify each metabolite that changes significantly in the global metabolite pool, and to assess the human (endogenous) biochemical responses to exposure to these exogenous (xenobiotic) compounds.

The complicated metabolic fate of chemicals in the human body is primarily determined by the chemical's structure and greatly varies according to dose, routes of exposure, inter- and intraindividual genetic differences, gut microbiota, diet, life style, and environment, as well as other xenobiotics intentionally or unintentionally present. In order to simplify these intricate factors, a knowledgebased engine that sequentially manipulates each factor in silico for prediction, coupled with in vitro screening and identification, and in vivo validation is deployed for xenobiotic metabolism studies [8]. However, because cross-platform extrapolations are not always the reliable or robust [9], the in vivo xenobiotic metabolite studies require special attention; these compounds are often intermixed against the background of endogenous metabolites in biological samples. Recent advances in liquid, and gas chromatography (LC and GC) and capillary electrophoresis (CE) coupled with mass spectrometry (LC-MS, GC-MS, and CE-MS) and nuclear magnetic resonance (NMR) have made it possible to simultaneously detect various small-molecule metabolites [10].

Metabolomics is the study of the metabolome, the complete set of endogenous metabolites in a biological sample. In context of systems biology [11, 12], metabolomics is favored not only for decoding how a biological system responds to a stimulus by identifying the most significantly affected endogenous metabolites and their metabolic pathways, but also resolving the relationships between endogenous and xenobiotic metabolic processes [13]. Metabolomics techniques have offered xenobiotic studies a novel way to differentiate the exogenous from the endogenous by untangling the interactive metabolic pathways for both. Capable of simultaneously analyzing hundreds and thousands of variables, metabolomics is uniquely suited to develop a new generation of PK platforms that can measure multiple nutraceutical components in vivo, as well as identify characteristic metabolic alterations in humans exposed to nutraceuticals [14, 15]. Along these lines, utilizing metabolomics to study herbal medicine efficacy and toxicity has been a key focus of recent herbal and pharmaceutical research [16-20]. Recently, we proposed an integrated metabolomic profiling strategy for PK and PD studies of multi-component drugs using tandem mass spectrometry (MS/MS) [21] and conducted a proof-ofconcept poly-PK study of $\mathrm{Pu}$-erh tea intervention in humans based on metabolomics [6].

Metabolomics might assume the new mission to model the variations of both exogenous and endogenous metabolites through a given biological system, which has vital implications in both systems biology and multi-component herbal medicine. The aim of this review is to discuss recent progress in the characterization of poly-PK and metabolism of multi-component xenobiotic agents such as compound drugs, dietary supplements, and herbal medicines using metabolomics. We also propose a research framework that integrates the dynamic concentration profile of bioavailable xenobiotic molecules that result from in vivo absorption, and hepatic and gut bacterial metabolism, as well as the human metabolic response profile.

\section{Metabolomics in Xenobiotic Intervention Study}

Metabolomics enables the profiling of a vast number of smallmolecule metabolites in an organism or a biological system as a whole, and helps understand how these metabolites respond to a stimulus, highlighting the most relevant potential biomarkers. When the stimulus is a xenobiotic intervention, metabolomics techniques are employed to profile the metabolite pool with and without the xenobiotic and highlight the differential variables to identify the xenobiotic metabolites. A comparison of a typical protocol for xenobiotic intervention studies using LC-MS-based metabolomics and the classical LC-MS-based workflow is illustrated in Figure 1. The major difference between the two methods is the data processing procedure to differentiate exogenous metabolites against the endogenous metabolite background. The metabolomics strategy employs multivariate data pretreatment methods [22] (e.g., deconvolution, alignment, integration) coupled with multivariate analyses $[15,23]$ (e.g., principle component analysis (PCA), partial least squares discriminant analysis (PLS-DA)). In contrast, the classical strategy utilizes arbitrary endogenous background subtraction and/or knowledge-based mass defect filtering, which takes advantage of known mass changes between the parent ion and metabolite ions [24].

Such a metabolomics-based protocol was first proposed in 2003 by Plumb and colleagues in an attempt to find the in vivo metabolites of GSK-X, the structure of which was unknown to the investigators [29]. Shortly after, another metabolomics-based protocol identified several new metabolites of citalopram [30]. Consequently, Dr. Gonzalez from National Cancer Institute at the National Institute of Health expanded LC-MS-based metabolomics for xenobiotic metabolism studies by developing various strategies, including (1) identification of metabolites by metabolomic analysis with or without xenobiotic intervention (Figure 1, and [31]), (2) validation of metabolites through metabolomic comparison between xenobiotic treatments with or without stable isotope label in order to highlight the xenobiotic metabolites in contrast to the endogenous ones altered by xenobiotic intervention, (3) identification of metabolic pathways through metabolomic comparison of wild-type and genetically-modified mice, and (4) identification of polymorphisms of genes encoding human xenobiotic metabolism enzymes (XMEs) that may be responsible for adverse drug reactions [31]. Two recent reviews address the technical issues and progress in this field $[32,33]$. 


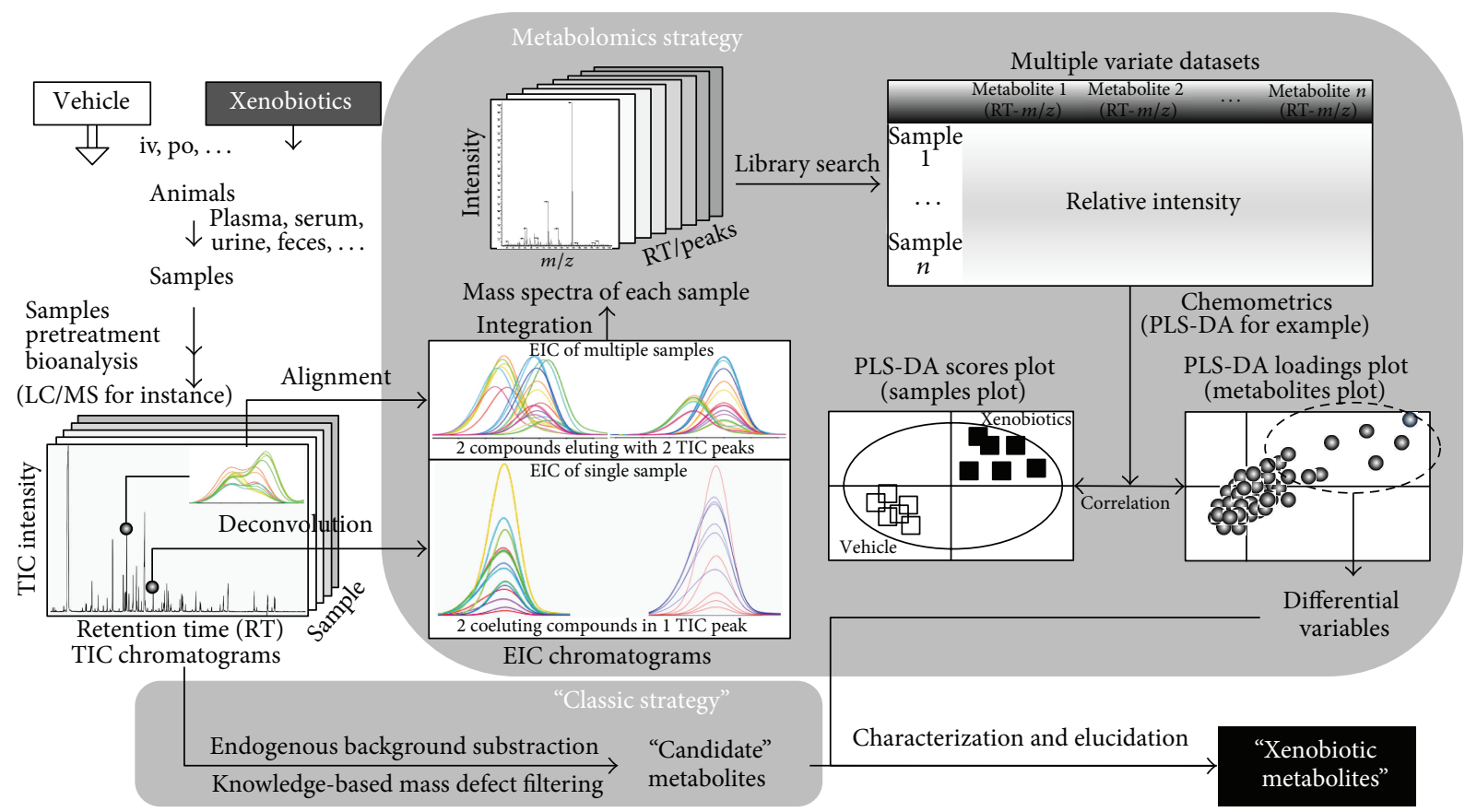

FIGURE 1: LC-MS protocol for xenobiotic intervention studies with and without the incorporation of metabolomics. Iv: intravenous; po: oral; EIC: extracted ion chromatogram; PLS-DA: partial least squares-discriminant analysis; TIC: total ion chromatogram.

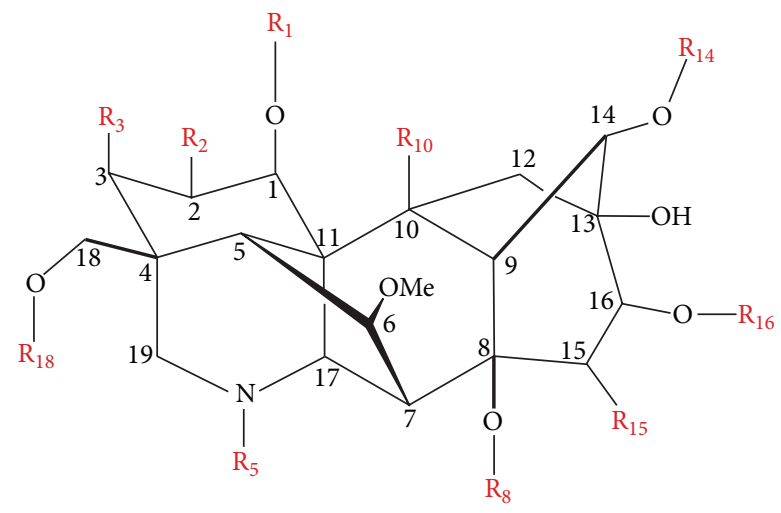

Major aconitum alkaloids in aconite roots

$\begin{array}{llllcllllll} & \mathrm{R}_{1} & \mathrm{R}_{2} & \mathrm{R}_{3} & \mathrm{R}_{5} & \mathrm{R}_{8} & \mathrm{R}_{10} & \mathrm{R}_{14} & \mathrm{R}_{15} & \mathrm{R}_{16} & \mathrm{R}_{18} \\ \text { Aconitine } & \mathrm{Me} & \mathrm{H} & \mathrm{OH} & \mathrm{Et} & \mathrm{Ac} & \mathrm{H} & \mathrm{Bz} & \mathrm{OH} & \mathrm{Me} & \mathrm{Me} \\ \text { Mesaconitine } & \mathrm{Me} & \mathrm{H} & \mathrm{OH} & \mathrm{Me} & \mathrm{Ac} & \mathrm{H} & \mathrm{Bz} & \mathrm{OH} & \mathrm{Me} & \mathrm{Me} \\ \text { Hypaconitine } & \mathrm{Me} & \mathrm{H} & \mathrm{H} & \mathrm{Me} & \mathrm{Ac} & \mathrm{H} & \mathrm{Bz} & \mathrm{OH} & \mathrm{Me} & \mathrm{Me} \\ \text { Jesaconitine } & \mathrm{Me} & \mathrm{H} & \mathrm{OH} & \mathrm{Et} & \mathrm{Ac} & \mathrm{H} & \mathrm{As} & \mathrm{OH} & \mathrm{Me} & \mathrm{Me} \\ \text { Yunaconitine } & \mathrm{Me} & \mathrm{H} & \mathrm{OH} & \mathrm{Et} & \mathrm{Ac} & \mathrm{H} & \mathrm{As} & \mathrm{H} & \mathrm{Me} & \mathrm{Me}\end{array}$

In vitro metabolites of aconitine \begin{tabular}{lllllllllll}
\multicolumn{1}{c}{$\mathrm{R}_{1}$} & $\mathrm{R}_{2}$ & $\mathrm{R}_{3}$ & $\mathrm{R}_{5}$ & $\mathrm{R}_{8}$ & $\mathrm{R}_{10}$ & $\mathrm{R}_{14}$ & $\mathrm{R}_{15}$ & $\mathrm{R}_{16}$ & $\mathrm{R}_{18}$ \\
Aconitine & $\mathrm{Me}$ & $\mathrm{H}$ & $\mathrm{OH}$ & $\mathrm{Et}$ & $\mathrm{Ac}$ & $\mathrm{H}$ & $\mathrm{Bz}$ & $\mathrm{OH}$ & $\mathrm{Me}$ & $\mathrm{Me}$ \\
In vitro rat CYP450 (Wang et al. [25]) & & & & &
\end{tabular}

$\begin{array}{llllllllllllllll}\text { M1 Me } & \mathrm{H} & \mathrm{OH} & \mathrm{Et} & \mathrm{Ac} & \mathrm{H} & \mathrm{Bz} & \mathrm{OH} & \mathrm{H} & \mathrm{Me}\end{array}$

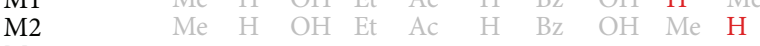
$\begin{array}{lllllllllllll}\text { M3 } & \mathrm{Me} & \mathrm{H} & \mathrm{OH} & \mathrm{Et} & \mathrm{Ac} & \mathrm{H} & \mathrm{Bz} & \mathrm{OH} & \mathrm{H} & \mathrm{H}\end{array}$ $\begin{array}{lllllllllll}\text { M4 } & \text { Me } & \mathrm{H} & \mathrm{OH} & \mathrm{Et} & \mathrm{H} & \mathrm{H} & \mathrm{Bz} & \mathrm{OH} & \mathrm{Me} & \mathrm{Me} \\ \text { M5 } & \mathrm{Me} & \mathrm{H} & =\mathrm{O} & \text { Et } & \mathrm{Ac} & \mathrm{H} & \mathrm{Bz} & \mathrm{OH} & \mathrm{Me} & \mathrm{Me}\end{array}$ $\begin{array}{lllllllllll}\text { M5 } & \mathrm{Me} & \mathrm{H} & \mathrm{O} & \mathrm{Et} & \mathrm{Ac} & \mathrm{H} & \mathrm{Bz} & \mathrm{OH} & \mathrm{Me} & \mathrm{Me} \\ \mathrm{M} 6 & \mathrm{Me} & \mathrm{H} & \mathrm{OH} & \mathrm{H} & \mathrm{Ac} & \mathrm{H} & \mathrm{Bz} & \mathrm{OH} & \mathrm{Me} & \mathrm{Me}\end{array}$ In vitro human intestinal bacteria (Zhao et al. [26]) $\begin{array}{lllllllllll}\text { M1, M4 } & & & & & & & & & & \\ \text { M7 } & \text { Me } & \mathrm{H} & \mathrm{H} & \text { Et } & \mathrm{Ac} & \mathrm{H} & \mathrm{Bz} & \mathrm{OH} & \mathrm{Me} & \mathrm{Me}\end{array}$ $\begin{array}{lllllllllll}\text { M8 } & \text { Me } & \mathrm{H} & \mathrm{H} & \mathrm{Et} & \mathrm{Ac} & \mathrm{H} & \mathrm{Bz} & \mathrm{OH} & \mathrm{H} & \mathrm{Me}\end{array}$ M9 Me $\mathrm{H}$ OH Et $\mathrm{R} \quad \mathrm{H} \quad \mathrm{Bz} \quad \mathrm{OH}$ Me Me R for M9: more than 20 kinds of esterification products with fat acids. In vitro rat intestinal bacteria (Wang et al. [28]) M4, M7, M9

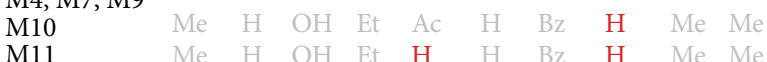
$\begin{array}{lllllllllllll}\text { M11 Me } & \mathrm{H} & \mathrm{OH} & \mathrm{Et} & \mathrm{H} & \mathrm{H} & \mathrm{Bz} & \mathrm{H} & \mathrm{Me} & \mathrm{Me}\end{array}$ M12 Me H OH Et Ac OH Bz OH Me Me

R for M9: octenoyl, hexenoyl, propionyl, butyryl, and 3-hydroxy-butyryl. In vitro human CYP450 (Tang et al. [27])

M1, M5, M6.

M13

M14

M15

FIGURE 2: Major aconitum alkaloids in aconite roots and in vitro metabolites of aconitine [25-28]. Me: methyl group; Et: ethyl group; Ac: acetyl group; Bz: benzoyl group; As: anisoyl group.

Compared to the classical methods in xenobiotic intervention studies, metabolomics strategies have clear advantages, firstly, in the capacity of handling a great number of variables-which allows a shift in purpose from targeting xenobiotic metabolites to profiling the complete set of metabolites in biological samples-and secondly, in the unbiased selection of variables that are significantly alteredwhich facilitates the discrimination of xenobiotic metabolites from endogenous ones. Due to these advantages of metabolomic strategies, a novel, evidence-based methodology that starts with mapping in vivo metabolites and leads to further in vitro characterization has been established, which is in stark contrast to the classic, knowledge-based methodology that starts with in vitro identification to in vivo characterization, and is frequently muddled by inconsistent results. Such a methodological shift obviates potential falsenegative results that usually result from traditional empirical strategies, and significantly deepens our insight into the in 

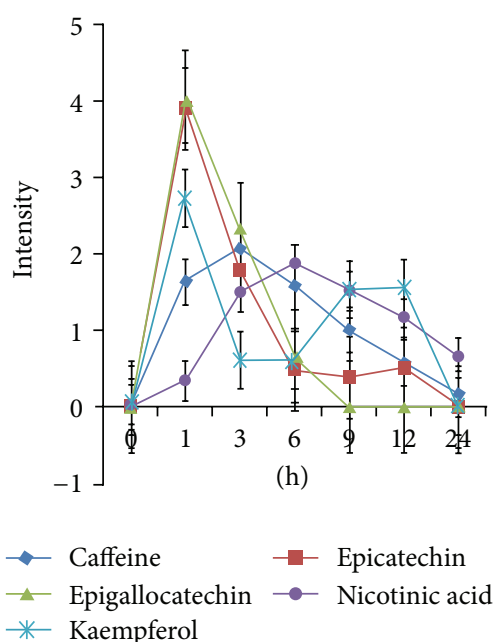

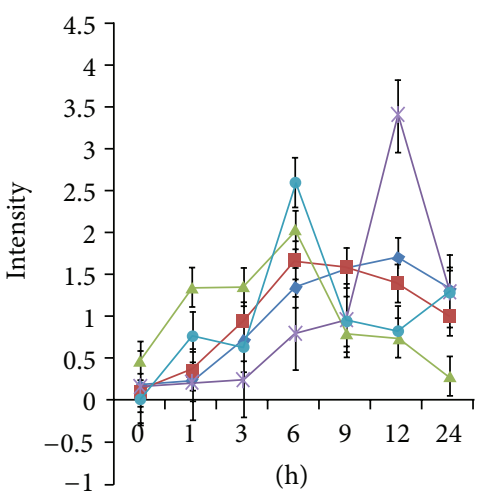

$\neg$ 1-Methyluric acid

- Hippuric acid

- 7-Methylhypoxanthine

- 1,7-Methyluric acid

* 2-Hydroxybenzoic acid

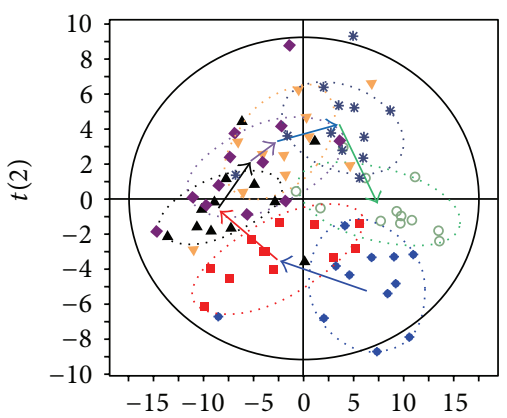

$t(1)$

$\begin{array}{ll}* 0 \mathrm{~h} & \nabla 9 \mathrm{~h} \\ -1 \mathrm{~h} & * 12 \mathrm{~h} \\ \text { - } 3 \mathrm{~h} & \circ 24 \mathrm{~h} \\ \text { - } 6 \mathrm{~h} & \end{array}$

(a)
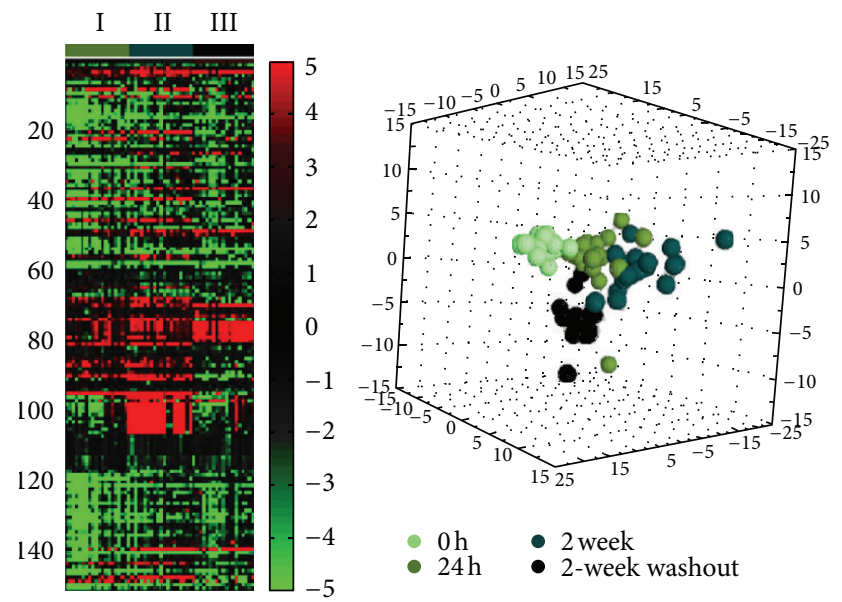

(b)

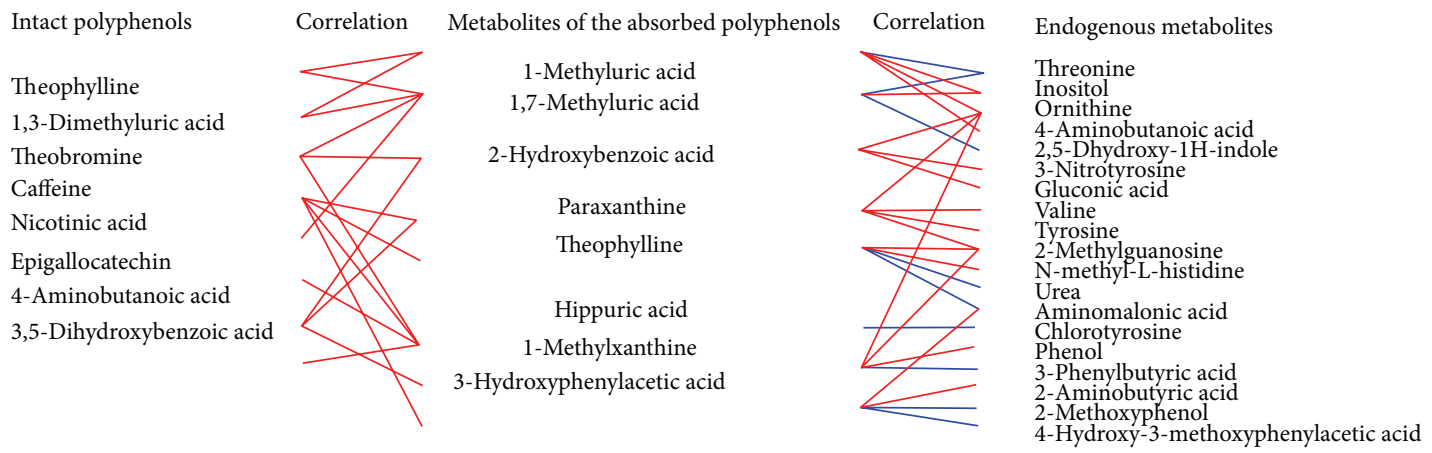

(c)

FIGURE 3: Dynamic concentration profile of bioavailable Pu-erh tea polyphenols due to in vivo absorption, and hepatic and gut bacterial metabolism, as well as the human metabolic response profile. (a) Urine concentration-time course of some representative substances, intact polyphenols, metabolites of the absorbed polyphenols, and altered endogenous metabolites, after Pu-erh tea intake; (b) effect of Pu-erh tea intake on human urine metabolite endpoints. (left panel) Heatmap showing differences in altered endogenous metabolites detected from the metabolome after $\mathrm{Pu}$-erh tea intake (postdose) as compared to predose metabolome. (I) metabolomic changes at $24 \mathrm{~h}$ postdose relative to predose; (II) 2-week postdose versus predose; (III) 2-week washout versus predose. Each cell in the heat map represents the fold change between the two time points (e.g., postdose versus the predose) for a particular metabolite. (right panel) 3D PCA scores plot of urinary metabolic profiles at predose, $24 \mathrm{~h}$ postdose, 2 week postdose, and 2 week washout postdose; (c) correlation of intact polyphenols, metabolites of the absorbed polyphenols, and altered endogenous metabolites in response to Pu-erh tea exposure. The relationships among the three groups of compounds were visualized in the form of correlation maps, which are displayed by red (positive) or blue (negative) lines. 


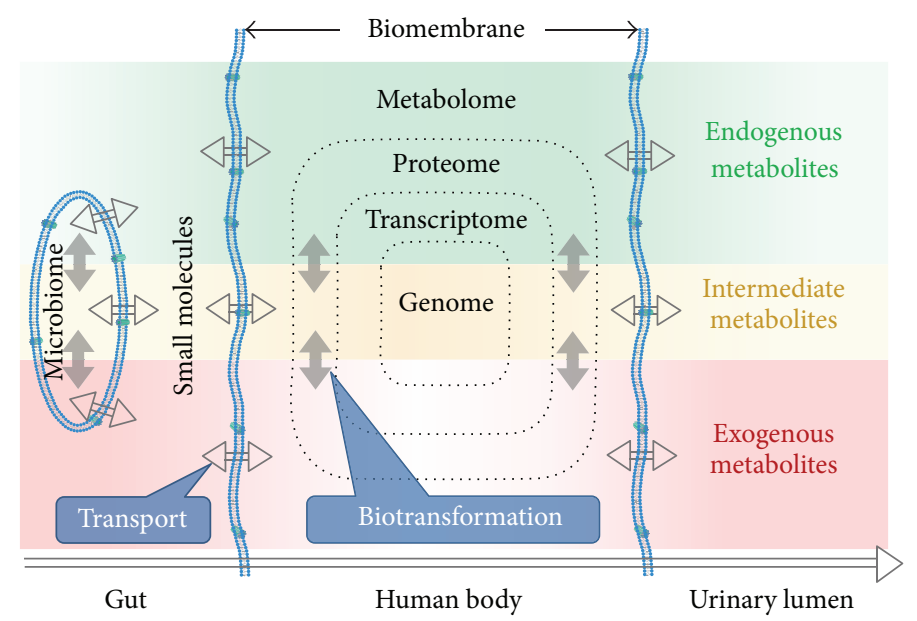

FIGURE 4: Research framework to integrate the dynamic concentration profile of bioavailable xenobiotic molecules due to in vivo absorption and the hepatic and gut bacterial metabolism, as well as the human metabolic response profile.

vivo metabolic map of xenobiotics in at least two ways. First, metabolomics captures in vivo xenobiotic metabolites that are otherwise easily overwhelmed by the endogenous background. Examples of such metabolites are those with unexpected skeleton biotransformation or those at extremely trace levels with potential clinical significance and wide usage in the general population. Second, the consideration of genetic and environmental factors affecting the metabolism of xenobiotics in metabolomics studies facilitates the in vivo identification of metabolic pathways and factors affecting them.

\section{Current Status of Single Xenobiotic Intervention Studies with Metabolomics Strategy}

To date, metabolomics techniques have been employed in metabolism studies of dozens of single xenobiotics with diverse chemical structures (Table 1), including GSK-X [29], citalopram [30], aminoflavone [34], arecoline/arecaidine [35], PhIP [36], arecoline 1-oxide [37], ferulic and sinapic acids [38], acetaminophen [39-42], dextromethorphan [43], melatonin [44, 45], vitamin E [46], fenofibrate [47, 48], tolcapone [49], cyclophosphamide/ifosfamide [50], tipranavir [51], nefazodone [52], valproic acid [53], ritonavir [54], pulegone/clozapine [42], thioTEPA [55], isoliquiritigenin [56], ethanol [57], and procainamide [58]. Remarkably, majority of these studies were carried out in vivo. It has been proposed that such metabolomics strategies can be used to study any organic molecule in any animal model, provided that the protocol is carefully designed, controlled, and executed [32]. In our opinion, a metabolomics approach is especially useful for studying xenobiotics with known toxicities and multiple metabolite endpoints predicted by their chemical structures. As a result, the insights from metabolomics extend our knowledge of how a xenobiotic affects human body from a list of its major metabolites to the complete in vivo metabolite map. The detailed information about multiple bioactive metabolites may significantly advance our understanding of various and rare adverse drug reactions in the general population.

\section{Progress and Challenges of Herbal Exposure Studies}

Multicomponent drugs and herbal medicines have an extremely complicated and highly variable chemical composition and introduce multiple xenobiotics into the human body. The metabolism of these xenobiotics may provide insights as to why and how they work by revealing changes in vivo due to herbal exposure. However, the unclear metabolic fate of herbal medicines causes significant limitations in understanding the efficacy and toxicity of these substances. Compared to single xenobiotics, of which there are significant advances in terms of metabolic fate, multi-component herbal medicines present a challenge due to their complex nature.

For the development of in vivo xenobiotic metabolism protocols for multi-component drugs and herbal medicines, there are several major obstacles: (1) the difficulty to determine and standardize the chemical composition of multicomponent drugs and herbal medicines, (2) the overlap between the chemical composition of herbal medicines with that of daily diets, (3) the extensive microbial-mammalian cometabolism in the gut of herbal components [67] that vary by species (e.g., see the in vitro metabolites of aconitine, illustrated in Figure 2 [25-28]), (4) the difficulty of differentiating exogenous metabolites from the endogenous background 1-3, and (5) the difficulty of resolving the intercrossed metabolic pathways of different herbal components that share similar chemical skeletons (for an example, see aconitum alkaloids in aconite roots, illustrated in Figure 2). Therefore, current research to elucidate the xenobiotic metabolism of herbal exposures is still in its infancy, exploring and developing solutions to these challenges. 
TABLE 1: Summary of PK studies of single chemicals and multicomponent drugs or herbal medicines using metabolomics.

\begin{tabular}{|c|c|c|c|c|}
\hline Substance & Type of biological sample & Bioanalysis technique & $\begin{array}{l}\text { Multivariate analysis } \\
\text { method }\end{array}$ & Reference \\
\hline GSK-X & Rat urine & LC-TOFMS & PCA, Ward's HCA & {$[29]$} \\
\hline Citalopram & Rat urine & LC-MS & $\begin{array}{c}\text { PCA, PLS, PARAFAC, } \\
\text { N-PLS }\end{array}$ & {$[30]$} \\
\hline Ferulic acid and sinapic acid & Rat urine & HPLC-QTOFMS & PLS-DA & {$[38]$} \\
\hline Fenofibrate & Rat urine & UPLC-QTOFMS & PLS-DA & {$[47]$} \\
\hline Isoliquiritigenin & Rat urine & UPLC-QTOFMS & PCA, PLS-DA & {$[56]$} \\
\hline Tolcapone & Rat urine & UPLC-QTOFMS & PCA & [49] \\
\hline Acetaminophen & Rat urine & UPLC/MS, NMR & PCA, PLS-DA & {$[41]$} \\
\hline Valproic acid & Mouse urine & LC-MS & PCA & {$[53]$} \\
\hline ThioTEPA & Mouse urine & UPLC-QTOFMS & OPLS & {$[55]$} \\
\hline NSC686288 (aminoflavone) & Mouse urine & UPLC-QTOFMS & PCA & {$[31,34]$} \\
\hline Ethanol & Mouse urine & UPLC-QTOFMS & PLS-DA, OPLS & [57] \\
\hline Arecoline and arecaidine & Mouse urine & UPLC-QTOFMS & PCA, PLS-DA & {$[35]$} \\
\hline$( \pm)$-Arecoline 1-oxide & Mouse urine & UPLC-QTOFMS & PCA & {$[37]$} \\
\hline $\mathrm{PhIP}^{*}$ & Mouse urine & UPLC-QTOFMS & PCA & {$[36]$} \\
\hline Acetaminophen & Mouse urine & UPLC-QTOFMS & PCA & {$[39]$} \\
\hline Cyclophosphamide/ifosfamide & Mouse urine & UPLC-QTOFMS & OPLS & {$[50]$} \\
\hline Vitamin E & Mouse urine & UPLC-TOFMS & PCA, PLS-DA & {$[46]$} \\
\hline Melatonin & Mouse urine & $\begin{array}{l}\text { IPLC-TOFMS, } \\
\text { LC-MS/MS }\end{array}$ & PCA, OPLS & {$[44]$} \\
\hline Tipranavir & Mouse urine, feces, tissue & UPLC-TOFMS & PCA, OPLS-DA & {$[51]$} \\
\hline Ritonavir & Mouse urine and feces & UPLC-TOFMS & OPLS-DA & {$[54]$} \\
\hline Procainamide & Mouse and human urine & UPLC-QTOFMS & PCA, PLS-DA & {$[58]$} \\
\hline Fenofibrate & Monkey urine & UPLC-QTOFMS & PLS-DA & {$[48]$} \\
\hline Pulegone/clozapine & Mouse liver & UPLC-TOFMS & PCA, OPLS-DA & {$[42]$} \\
\hline Dextromethorphan & Human urine & LC-MS/MS & PCA, OPLS-DA & {$[43]$} \\
\hline 3,4-Dehydro-debrisoquine & Human urine & UPLC-QTOFMS & OPLS & {$[31,59]$} \\
\hline Cocoa powder & Human urine & HPLC-QTOFMS & PLS-DA, two-way HCA & {$[60]$} \\
\hline Almond skin extract & Human urine & LC-QTOFMS & PCA, OPLS-DA & {$[61]$} \\
\hline Pu-erh tea & Human urine & $\begin{array}{l}\text { UPLC-QTOFMS, } \\
\text { GC-TOFMS }\end{array}$ & PCA, OPLS-DA & {$[6,62]$} \\
\hline $\begin{array}{l}\text { A mix of wine extract and grape } \\
\text { juice extract }\end{array}$ & Human urine & ${ }^{1} \mathrm{H}$ NMR & ANOVA-PCA/PLS & {$[63]$} \\
\hline $\begin{array}{l}\text { Dried black tea extract and red } \\
\text { grape extract }\end{array}$ & Human urine & ${ }^{1} \mathrm{H}$ NMR & ANOVA-PCA/PLS & {$[64]$} \\
\hline Chamomile tea & Human urine & ${ }^{1} \mathrm{H}$ NMR & PCA, PLS, OSC & {$[14]$} \\
\hline Dark chocolate & Human urine, plasma & $\begin{array}{l}{ }^{1} \mathrm{H} \text { NMR, LC-MS, } \\
\text { LC-MS/MS }\end{array}$ & PCA, OPLS-DA & {$[65]$} \\
\hline Nefazodone & $\begin{array}{l}\text { NADPH-supplemented } \\
\text { human liver microsomal } \\
\text { incubation samples }\end{array}$ & LC-MS & PCA & {$[52]$} \\
\hline $\begin{array}{l}\text { Rifampicin, phenobarbital, and } \\
\text { CITCO }^{* *}\end{array}$ & Human hepatocytes & UPLC-TOFMS & PCA, OPLS, SUS plot & {$[66]$} \\
\hline
\end{tabular}

*2-Amino-1-methyl-6-phenylimidazo[4,5-b]pyridine.

** 6-(4-Chlorophenyl)imidazo[2,1-b][1,3] thiazole-5-carbaldehydeO-3,4-dichlorobenzyl) oxime.

LC-MS: liquid chromatography-mass spectrometry; UPLC-QTOFMS: ultraperformance liquid chromatography-quadrupole time of flight mass spectrometry; NMR: nuclear magnetic resonance; GC-TOFMS: gas chromatography-time of flight mass spectrometry; PCA: principle component analysis; PLS: partial least squares; OPLS-DA: orthogonal partial least squares-discriminant analysis; DAPARAFAC: parallel factor analysis; N-PLS: multilinear partial least squares; OSC: orthogonal signal correction. 
4.1. Classic Strategy. The strategies and techniques for in vitro and in vivo herbal exposure studies following classic, knowledge-based methodologies have recently been discussed $[68,69]$. With the advances in bioanalytical methods, many metabolites of herbal interventions were successfully captured. For example, a recent study of the herbal supplement Danggui Buxue Tang detected and identified 142 metabolites from bile and plasma samples [70]. However, from the efforts to map the metabolites of single xenobiotics, it has become clear that the predictive and provisional approaches used in classic strategies can be inaccurate and lack reproducibility.

Nevertheless, two strategies have accomplished noteworthy advances in metabolite mapping of xenobiotics. One of these strategies was developed from the identification of individual metabolites of licorice [71]. In fact, more than 60 metabolites were identified and PK profiles of 55 were obtained [72]. This strategy was robust and managed to reveal intraherb metabolic interactions; however, it requires prior knowledge of herbal components and is time consuming. The other strategy was based on matching the "chemicalome" (referring to LC-MS data of the tested herbal medicine) to the "metabolome" (referring to LC-MS data including tentative herbal metabolites acquired by endogenous background subtraction) upon the injection of the herbal medicine Mailuoning [73]. Matching was performed by mass defect filtering, which incorporated accurate mass changes of 62 types of metabolic reactions. Using this approach, 143 metabolites were identified in urine. This strategy exhibited clear superiority in labor-saving predictions; however, the endogenous background subtraction was arbitrary, introducing false positives and excluding false negative results.

4.2. Metabolomics-Based Strategy. The Recent advances in metabolomics encouraged researchers to start exploring the effects of multi-component drugs/herbal medicines using metabolomics strategies. To the best of our knowledge, the first attempt of this kind was the NMR-based metabolomics study on human nutritional intervention of grape/wine extract reported by van Velzen and colleagues in 2008 [63]. This study aimed to distinguish between-subject and within-subject variations in metabolomic data matrices using multivariate paired data analyses corresponding to a crossover design. Since its major results concerned metabolites of polyphenols, the integration of PK modeling into metabolomics was developed in a later study by the same group [64]. The proposed multivariate paired data analysis [74] and dynamic metabolomic data analysis [75], in combination with study designs that tackle the challenges arising from dietary metabolite background and microbial-human cometabolism in the gut [76], have collectively coined the term "nutrikinetics," a new discipline that combines cuttingedge technologies and new methodologies to get a complete picture of what happens to the consumed metabolites and supplements in the human body [77]. Nutritkinetics relies heavily on metabolomics biostatistical analyses. To date, there have been a number of studies on dietary exposure incorporating metabolomics, which were summarized in a recent review on nutrimetabolomic strategies [78].
The metabolomics-based strategies using LC-MS have clear advantages, as they can handle a great number of variables and large datasets, enable graphic representation of metabolism-related sample classification, and identify drug compounds and significant drug metabolites. In fact, several publications reported the use of metabolomics to capture both drug-derived and drug-induced metabolites [66] and the alterations of metabolites in urine after cocoa powder consumption [60] (Table 1). Despite the fact that metabolomics can be employed in PK studies of herbal medicines, it is almost impossible to detect the complete pool of metabolites in a biological sample the existing analytical platforms [79]. Indeed, categorizing the origins of altered metabolites as a result of herbal intervention still present with significant challenges [21], including the categorization of: (1) intact herbal components absorbed into circulation, (2) xenobiotic metabolites processed by hepatic enzymes and gut microbes, and (3) endogenous metabolites that are significantly altered in response to the intake of plant-derived compounds.

We believe that categorization of these metabolites in herbal intervention studies will benefit more from developments in multivariate analyses than in advances in analytical techniques. To this end, we have proposed a multivariate similarity analysis to categorize the altered metabolites as a result of herbal intervention [21]. Recently, we used this strategy to analyze the metabolic fates of Pu-erh tea polyphenols in humans [6]. Urine samples were collected at baseline and at different time periods for 6 weeks. Volunteers ingested $\mathrm{Pu}$-erh tea daily, followed by a wash-out phase during these 6 weeks. The urine samples were analyzed using ultraperformance liquid chromatography-quadrupole time of flight mass spectrometry (UPLC-QTOFMS) and gas chromatography-time of flight mass spectrometry (GCTOFMS). The resulting dataset composed of 6,028 detected features was subjected to univariate statistical analysis, yielding 2,476 and 176 altered variables from UPLC-QTOFMS and GC-TOFMS were highlighted, respectively $(P<0.05)$. Using multivariate similarity analysis to compare the altered variables to the plant metabolome or the predose human metabonome, 19, 26, and 118 metabolites were categorized as intact tea polyphenols, metabolites of the absorbed polyphenols, and endogenous metabolites altered due to tea intake, respectively. The subsequent dynamic correlation analysis produced, for the first time, a correlation between the herbal metabolic network and endogenous metabolism (Figure 3).

As discussed above, despite great challenges, a strategy based on metabolomics has great potential capabilities to discover not only the components in herbal exposure and their in vivo metabolites, but also the endogenous metabolites altered by herbal interventions. In this regard, the metabolic shells covering the interactions between multiple xenobiotics and human biological system will be shucked in future metabolomics incorporating xenobiotic metabolism.

\section{Proposed Research Framework}

The essence of balances in traditional Chinese medicine and herbal medicines align very closely with the core concepts 
of systems biology [80], which aims to theoretically and experimentally describe homeostasis of a biological system and its allostasis with an "-omics" approach [81, 82]. Metabolomics, as a "top-down" -omics technology, evaluating small-molecule metabolites as the ultimate downstream products of genomic, transcriptomic, and/or proteomic perturbations, is encouraging herbal medicine researchers to tackle existing issues in the field from chemistry to biology. However, besides its phytochemical applications, which focus on the quality assessments of botanic products [8385], metabolomics in herbal medicine mostly addresses biomedical implications for the endogenous metabolism [8690], while paying little attention to the metabolism of the xenobiotics, which is tightly linked to and has important implications for human metabolic networks.

Based on the above and guidance from related studies, we propose a research framework to integrate the dynamic concentration profile of bioavailable xenobiotic molecules due to in vivo absorption and the hepatic and gut bacterial metabolism, as well as the human metabolic response profile in Figure 4. Our framework highlights the flux of smallmolecule metabolites through the human body with gut as the major entrance and urinary lumen as the major exit points. Recent multicompartmental (plasma, urine, and caecal contents) metabolomic work depicted this framework in a smaller scale [91]. In our proposed model, the human body has evolved as a superorganism encompassing the genome, transcriptome, proteome, metabolome, and symbiotic gut microbiome, that latter coexisting with the host depending on genetic and environmental factors [92-96]. It is of special importance for the gut microbiome and the host to maintain ceaseless substance exchange, in which the small-molecule metabolites can permeate the biomembrane barrier. These metabolites can be divided into endogenous and exogenous metabolites according to their predicted sources; however, the distinction between endogenous and exogenous is not so clear due to the gut microbiome. As a result, metabolites with unclear origins can be classified in an intermediate group. For example, endogenous ethanol is not a metabolite of human metabolism, but a byproduct of gut microbes $[97,98]$, which is associated with nonalcoholic fatty liver disease [99]. In all, biotransformation (metabolism) and transportation are essential to the metabolic network of host and microbial interactions to dispose of endogenous and exogenous metabolites.

The host-gut microbial metabolic networks for endogenous and exogenous metabolites are highly interactive but with subtle differences in metabolic fates. It is beyond doubt that the superorganism's metabolism has to strike a balance between the uptake of essential nutrients and the elimination of superfluous metabolites to maintain homeostasis, yielding a relatively stable internal environment of endogenous metabolites. The involvement of gut microbiota in endogenous metabolic pathways [100-104], as a matter of fact, has given rise to interactive host-microbiota metabolic, signaling, and immuneinflammatory axes that physiologically connect the gut, liver, muscle, and brain [105]. On the other hand, the superorganism cannot be too lenient with regard to exogenous metabolites, maintaining allostasis to achieve a stable internal environment by eliminating exogenous metabolites. Gut microbiota supplements the human metabolic system with xenobiotics [106] by directly taking part in their metabolism, especially in the metabolism of natural products such as flavonoids, saponins [67], polyphenols [76], and alkaloids [26, 28], as well as the metabolism of exogenous metabolites, such as the competitive sulfation of acetaminophen and p-cresol [107], and the regulation of XMEs [108, 109]. Based on the distinction between the metabolic fates of exogenous and endogenous metabolites, trend analysis was proposed as an alternative to metabolite discovery [110].

Therefore, the superorganism has evolved a highly delicate and interactive host-gut microbiota metabolic network to keep its homeostasis and trigger allostasis. Following our framework, in combination with advances in genomics, transcriptomics, proteomics, and metagenomics, future metabolomics strategies will contribute to our understanding of how the balance emphasized in comprehensive herbal medicine is correlated with the homeostasis and allostasis addressed in systems biology.

\section{Conclusion}

In conclusion, recent studies suggest that metabolomics coupled with multivariate statistical tools can offer an alternative to address the challenges of the determination of PK of multi-component pharmaceuticals and/or nutraceuticals. We believe that the use of a metabolomics strategy in pharmacological studies has important advantages over conventional approaches for multi-component therapeutics. The integration of metabolomics to study the metabolism of xenobiotics will unravel the complicated variations in multiple metabolites of endogenous and exogenous origin within the host-gut microbial symbiotic network, and tease out the underlying mechanisms of homeostasis and allostasis in terms of systems biology. Progress in this field will definitely help the poly-PK studies of multi-component herbal medicines to assess their efficacy. Acquisition of a complete and dynamic panel of pharmacokinetic parameters for multicomponent dosage regimens to achieve desired therapeutic efficacies is essential to minimize toxicity, reduce overdosing and drug complications, keep healthcare costs at a minimum, and ultimately, increase patient compliance and quality of life.

\section{References}

[1] R. L. Blaylock, "New developments in phytoprevention and treatment of cancer," The Journal of the American Nutraceutical Association, vol. 2, pp. 19-29, 1999.

[2] J. M. Hollander and J. I. Mechanick, "Complementary and alternative medicine and the management of the metabolic syndrome," Journal of the American Dietetic Association, vol. 108, no. 3, pp. 495-509, 2008.

[3] K. Chan, "Progress in traditional Chinese medicine," Trends in Pharmacological Sciences, vol. 16, no. 6, pp. 182-187, 1995.

[4] G. W. Plumb, M. T. Garcia-Conesa, P. A. Kroon, M. Rhodes, S. Ridley, and G. Williamson, "Metabolism of chlorogenic acid by 
human plasma, liver, intestine and gut microflora," Journal of the Science of Food and Agriculture, vol. 79, no. 3, pp. 390-392, 1999.

[5] S. M. He, E. Chan, and S. F. Zhou, "ADME properties of herbal medicines in humans: evidence, challenges andstrategies," Current Pharmaceutical Design, vol. 17, no. 4, pp. 357-407, 2011.

[6] G. Xie, A. Zhao, L. Zhao et al., "Metabolic fate of tea polyphenols in humans," Journal of Proteome Research, vol. 11, no. 6, pp. 3449-3457, 2012.

[7] T. Xue and R. Roy, "Studying traditional Chinese medicine," Science, vol. 300, no. 5620, pp. 740-741, 2003.

[8] A. E. F. Nassar and R. E. Talaat, "Strategies for dealing with metabolite elucidation in drug discovery and development," Drug Discovery Today, vol. 9, no. 7, pp. 317-327, 2004.

[9] O. Pelkonen, A. Tolonen, T. Korjamo, M. Turpeinen, and H. Raunio, "From known knowns to known unknowns: predicting in vivo drug metabolites," Bioanalysis, vol. 1, no. 2, pp. 393-414, 2009.

[10] A. Tolonen, M. Turpeinen, and O. Pelkonen, "Liquid chromatography-mass spectrometry in in vitro drug metabolite screening," Drug Discovery Today, vol. 14, no. 3-4, pp. 120-133, 2009.

[11] J. C. Lindon, E. Holmes, and J. K. Nicholson, "Metabonomics: systems biology in pharmaceutical research and development," Current Opinion in Molecular Therapeutics, vol. 6, no. 3, pp. 265272, 2004.

[12] J. K. Nicholson and J. C. Lindon, "Systems biology: metabonomics," Nature, vol. 455, no. 7216, pp. 1054-1056, 2008.

[13] J. K. Nicholson and I. D. Wilson, "Understanding "global" systems biology: metabonomics and the continuum of metabolism," Nature Reviews Drug Discovery, vol. 2, no. 8, pp. 668-676, 2003.

[14] Y. Wang, H. Tang, J. K. Nicholson, P. J. Hylands, J. Sampson, and E. Holmes, "A metabonomic strategy for the detection of the metabolic effects of chamomile (Matricaria recutita L.) ingestion," Journal of Agricultural and Food Chemistry, vol. 53, no. 2, pp. 191-196, 2005.

[15] L. W. Sumner, E. Urbanczyk-Wochniak, and C. D. Broeckling, "Metabolomics data analysis, visualization, and integration," Methods in Molecular Biology, vol. 406, pp. 409-436, 2007.

[16] G. Xie, R. Plumb, M. Su et al., "Ultra-performance LC/TOF MS analysis of medicinal Panax herbs for metabolomic research," Journal of Separation Science, vol. 31, no. 6-7, pp. 1015-1026, 2008.

[17] S. C. Wu, G. C. Yen, B. S. Wang et al., "Antimutagenic and antimicrobial activities of pu-erh tea," LWT-Food Science and Technology, vol. 40, no. 3, pp. 506-512, 2007.

[18] B. S. Wang, H. M. Yu, L. W. Chang, W. J. Yen, and P. D. Duh, "Protective effects of pu-erh tea on LDL oxidation and nitric oxide generation in macrophage cells," LWT-Food Science and Technology, vol. 41, no. 6, pp. 1122-1132, 2008.

[19] Y. Ni, M. Su, J. Lin et al., "Metabolic profiling reveals disorder of amino acid metabolism in four brain regions from a rat model of chronic unpredictable mild stress," FEBS Letters, vol. 582, no. 17, pp. 2627-2636, 2008.

[20] X. Q. Chen, Y. Ye, H. Cheng, J. F. Yin, and C. Sun, "Comparative analysis of theanine, caffein and polyphenolic constituents in green tea, black tea and puer tea," Food Research and Development, vol. 28, no. 12, pp. 141-144, 2007.

[21] K. Lan and W. Jia, "An Integrated metabolomics and pharmacokinetics strategy for multi-component drugs evaluation," Current Drug Metabolism, vol. 11, no. 1, pp. 105-114, 2010.
[22] X. Wei, X. Shi, S. Kim et al., "Data preprocessing method for liquid chromatography-mass spectrometry based metabolomics," Analytical Chemistry, vol. 84, no. 18, pp. 7963-7971, 2012.

[23] J. Trygg, E. Holmes, and T. Lundstedt, "Chemometrics in metabonomics," Journal of Proteome Research, vol. 6, no. 2, pp. 469-479, 2007.

[24] S. Ma and S. K. Chowdhury, "Analytical strategies for assessment of human metabolites in preclinical safety testing," Analytical Chemistry, vol. 83, no. 13, pp. 5028-5036, 2011.

[25] Y. Wang, S. Wang, Y. Liu, L. Yan, G. Dou, and Y. Gao, "Characterization of metabolites and cytochrome P450 isoforms involved in the microsomal metabolism of aconitine," Journal of Chromatography B, vol. 844, no. 2, pp. 292-300, 2006.

[26] Y. F. Zhao, F. R. Song, X. H. Guo, and S. Y. Liu, "Studies on the biotransformation of aconitine in human intestinal bacteria using soft-ionization mass spectrometry," Chemical Journal of Chinese Universities, vol. 29, no. 1, pp. 55-59, 2008.

[27] L. Tang, L. Ye, C. Lv, Z. Zheng, Y. Gong, and Z. Liu, "Involvement of CYP3A4/5 and CYP2D6 in the metabolism of aconitine using human liver microsomes and recombinant CYP450 enzymes," Toxicology Letters, vol. 202, no. 1, pp. 47-54, 2011.

[28] X. Wang, Z. Pi, W. Liu, Y. Zhao, and S. Liu, "Effect of pH on the metabolism of aconitine under rat intestinal bacteria and analysis of metabolites using HPLC/MS-MSn technique," Chinese Journal of Chemistry, vol. 28, no. 12, pp. 2494-2500, 2010.

[29] R. S. Plumb, C. L. Stumpf, J. H. Granger, J. Castro-Perez, J. N. Haselden, and G. J. Dear, "Use of liquid chromatography/timeof-flight mass spectrometry and multivariate statistical analysis shows promise for the detection of drug metabolites in biological fluids," Rapid Communications in Mass Spectrometry, vol. 17, no. 23, pp. 2632-2638, 2003.

[30] H. Idborg, P. O. Edlund, and S. P. Jacobsson, "Multivariate approaches for efficient detection of potential metabolites from liquid chromatography/mass spectrometry data," Rapid Communications in Mass Spectrometry, vol. 18, no. 9, pp. 944-954, 2004.

[31] C. Chen, F. J. Gonzalez, and J. R. Idle, "LC-MS-based metabolomics in drug metabolism," Drug Metabolism Reviews, vol. 39, no. 2-3, pp. 581-597, 2007.

[32] A. D. Patterson, F. J. Gonzalez, and J. R. Idle, "Xenobiotic metabolism: a view through the metabolometer," Chemical Research in Toxicology, vol. 23, no. 5, pp. 851-860, 2010.

[33] C. H. Johnson, A. D. Patterson, J. R. Idle, and F. J. Gonzalez, "Xenobiotic metabolomics: major impact on the metabolome," Annual Review of Pharmacology and Toxicology, vol. 52, pp. 3756, 2012.

[34] C. Chen, L. Meng, X. Ma et al., "Urinary metabolite profiling reveals CYP1A2-mediated metabolism of NSC686288 (aminoflavone)," Journal of Pharmacology and Experimental Therapeutics, vol. 318, no. 3, pp. 1330-1342, 2006.

[35] S. Giri, J. R. Idle, C. Chen, T. M. Zabriskie, K. W. Krausz, and F. J. Gonzalez, "A metabolomic approach to the metabolism of the areca nut alkaloids arecoline and arecaidine in the mouse," Chemical Research in Toxicology, vol. 19, no. 6, pp. 818-827, 2006.

[36] C. Chen, X. Ma, M. A. Malfatti et al., "A comprehensive investigation of 2-amino-1-methyl-6-phenylimidazo [4,5-b] pyridine (PhIP) metabolism in the mouse using a multivariate data analysis approach," Chemical Research in Toxicology, vol. 20, no. 3, pp. 531-542, 2007. 
[37] S. Giri, K. W. Krausz, J. R. Idle, and F. J. Gonzalez, "The metabolomics of $( \pm)$-arecoline 1-oxide in the mouse and its formation by human flavin-containing monooxygenases," Biochemical Pharmacology, vol. 73, no. 4, pp. 561-573, 2007.

[38] A. Fardet, R. Llorach, A. Orsoni et al., "Metabolomics provide new insight on the metabolism of dietary phytochemicals in rats," Journal of Nutrition, vol. 138, no. 7, pp. 1282-1287, 2008.

[39] C. Chen, K. W. Krausz, J. R. Idle, and F. J. Gonzalez, "Identification of novel toxicity-associated metabolites by metabolomics and mass isotopomer analysis of acetaminophen metabolism in wild-type and Cyp2el-null mice," Journal of Biological Chemistry, vol. 283, no. 8, pp. 4543-4559, 2008.

[40] J. Cheng, X. Ma, K. W. Krausz, J. R. Idle, and F. J. Gonzalez, "Rifampicin-activated human pregnane $\mathrm{X}$ receptor and CYP3A4 induction enhance acetaminophen-induced toxicity," Drug Metabolism and Disposition, vol. 37, no. 8, pp. 1611-1621, 2009.

[41] J. Sun, L. K. Schnackenberg, and R. D. Beger, "Studies of acetaminophen and metabolites in urine and their correlations with toxicity using metabolomics," Drug Metabolism Letters, vol. 3, no. 3, pp. 130-136, 2009.

[42] F. Li, J. Lu, and X. Ma, "Profiling the reactive metabolites of xenobiotics using metabolomic technologies," Chemical Research in Toxicology, vol. 24, no. 5, pp. 744-751, 2011.

[43] U. Lutz, N. Bittner, R. W. Lutz, and W. K. Lutz, "Metabolite profiling in human urine by LC-MS/MS: method optimization and application for glucuronides from dextromethorphan metabolism," Journal of Chromatography B, vol. 871, no. 2, pp. 349-356, 2008.

[44] X. Ma, C. Chen, K. W. Krausz, J. R. Idle, and F. J. Gonzalez, "A metabolomic perspective of melatonin metabolism in the mouse," Endocrinology, vol. 149, no. 4, pp. 1869-1879, 2008.

[45] S. Niu, F. Li, D. X. Tan et al., "Analysis of $N^{1}$-acetyl- $N^{2}$-formyl5-methoxykynuramine/ $N^{1}$-acetyl-5-methoxy-kynuramine formation from melatonin in mice," Journal of Pineal Research, vol. 49, no. 2, pp. 106-114, 2010.

[46] J. Y. Cho, D. W. Kang, X. Ma et al., "Metabolomics reveals a novel vitamin $\mathrm{E}$ metabolite and attenuated vitamin $\mathrm{E}$ metabolism upon PXR activation," Journal of Lipid Research, vol. 50, no. 5, pp. 924-937, 2009.

[47] A. Liu, Y. Chen, Z. Yang et al., "New metabolites of fenofibrate in SpragueDawley rats by UPLC-ESI-QTOF-MS-based metabolomics coupled with LC-MS/MS," Xenobiotica, vol. 39, no. 4, pp. 345-354, 2009.

[48] A. Liu, A. D. Patterson, Z. Yang et al., "Fenofibrate metabolism in the cynomolgus monkey using ultraperformance liquid chromatography-quadrupole time-of-flight mass spectrometry-based metabolomics," Drug Metabolism and Disposition, vol. 37, no. 6, pp. 1157-1163, 2009.

[49] J. Sun, L. S. Von Tungeln, W. Hines, and R. D. Beger, "Identification of metabolite profiles of the catechol-O-methyl transferase inhibitor tolcapone in rat urine using LC/MS-based metabonomics analysis," Journal of Chromatography B, vol. 877, no. 24, pp. 2557-2565, 2009.

[50] F. Li, A. D. Patterson, C. C. Höfer, K. W. Krausz, F. J. Gonzalez, and J. R. Idle, "Comparative metabolism of cyclophosphamide and ifosfamide in the mouse using UPLC-ESI-QTOFMS-based metabolomics," Biochemical Pharmacology, vol. 80, no. 7, pp. 1063-1074, 2010.

[51] F. Li, L. Wang, G. L. Guo, and X. Ma, "Metabolism-mediated drug interactions associated with ritonavir-boosted tipranavir in mice," Drug Metabolism and Disposition, vol. 38, no. 5, pp. 871-878, 2010.

[52] R. P. Schneider, H. Zhang, L. Mu, A. S. Kalgutkar, and R. Bonner, "Utility of multivariate analysis in support of in vitro metabolite identification studies: retrospective analysis using the antidepressant drug nefazodone," Xenobiotica, vol. 40, no. 4, pp. 262-274, 2010.

[53] J. Sun, L. K. Schnackenberg, D. K. Hansen, and R. D. Beger, "Study of valproic acid-induced endogenous and exogenous metabolite alterations using LC-MS-based metabolomics," Bioanalysis, vol. 2, no. 2, pp. 207-216, 2010.

[54] F. Li and X. Ma, "Metabolomic screening and identification of the bioactivation pathways of ritonavir," Chemical Research in Toxicology, vol. 24, no. 12, pp. 2109-2114, 2011.

[55] F. Li, A. D. Patterson, C. C. Höfer, K. W. Krausz, F. J. Gonzalez, and J. R. Idle, "A comprehensive understanding of thioTEPA metabolism in the mouse using UPLC-ESI-QTOFMS-based metabolomics," Biochemical Pharmacology, vol. 81, no. 8, pp. 1043-1053, 2011.

[56] G. Tan, Z. Lou, X. Dong, W. Li, and Y. Chai, "Urinary metabolites of isoliquiritigenin in wistar rats using UHPLCTOF-MS-based xenometabolomics," Chromatographia, vol. 74, no. 3-4, pp. 341-348, 2011.

[57] X. Shi, D. Yao, and C. Chen, "Identification of N-acetyltaurine as a novel metabolite of ethanol through metabolomics-guided biochemical analysis," Journal of Biological Chemistry, vol. 287, no. 9, pp. 6336-6349, 2012.

[58] F. Li, A. D. Patterson, K. W. Krausz et al., "Metabolomics reveals the metabolic map of procainamide in humans and mice," Biochemical Pharmacology, vol. 83, no. 10, pp. 1435-1444, 2012.

[59] Y. Zhen, O. Slanař, K. W. Krausz et al., "3,4-Dehydrodebrisoquine, a novel debrisoquine metabolite formed from 4-hydroxydebrisoquine that affects the CYP2D6 metabolic ratio," Drug Metabolism and Disposition, vol. 34, no. 9, pp. 1563-1574, 2006.

[60] R. Llorach-Asunción, O. Jauregui, M. Urpi-Sarda, and C. Andres-Lacueva, "Methodological aspects for metabolome visualization and characterization. A metabolomic evaluation of the $24 \mathrm{~h}$ evolution of human urine after cocoa powder consumption," Journal of Pharmaceutical and Biomedical Analysis, vol. 51, no. 2, pp. 373-381, 2010.

[61] R. Llorach, I. Garrido, M. Monagas et al., "Metabolomics study of human urinary metabolome modifications after intake of almond (Prunus dulcis (Mill.) D.A. Webb) skin polyphenols," Journal of Proteome Research, vol. 9, no. 11, pp. 5859-5867, 2010.

[62] G. Xie, M. Ye, Y. Wang et al., "Characterization of pu-erh tea using chemical and metabolic profiling approaches," Journal of Agricultural and Food Chemistry, vol. 57, no. 8, pp. 3046-3054, 2009.

[63] E. J. J. van Velzen, J. A. Westerhuis, J. P. M. van Duynhoven et al., "Multilevel data analysis of a crossover designed human nutritional intervention study," Journal of Proteome Research, vol. 7, no. 10, pp. 4483-4491, 2008.

[64] E. J. J. van Velzen, J. A. Westerhuis, J. P. M. van Duynhoven et al., "Phenotyping tea consumers by nutrikinetic analysis of polyphenolic end-metabolites," Journal of Proteome Research, vol. 8, no. 7, pp. 3317-3330, 2009.

[65] F. P. J. Martin, S. Rezzi, E. Peré-Trepat et al., "Metabolic effects of dark chocolate consumption on energy, gut microbiota, and stress-related metabolism in free-living subjects," Journal of Proteome Research, vol. 8, no. 12, pp. 5568-5579, 2009. 
[66] V. Croixmarie, T. Umbdenstock, O. Cloarec et al., "Integrated comparison of drug-related and drug-induced ultra performance liquid chromatography/mass spectrometry metabonomic profiles using human hepatocyte cultures," Analytical Chemistry, vol. 81, no. 15, pp. 6061-6069, 2009.

[67] H. Li, M. Zhou, A. Zhao, and W. Jia, "Traditional Chinese medicine: balancing the gut ecosystem," Phytotherapy Research, vol. 23, no. 9, pp. 1332-1335, 2009.

[68] P. Li, E.-H. Liu, and X.-D. Wen, "Analysis of Chinese herbal medicines with holistic approaches and integrated evaluation models," TrAC-Trends in Analytical Chemistry, vol. 27, no. 1, pp. 66-77, 2008.

[69] G.-Z. Xin, L.-W. Qi, Z.-Q. Shi, G.-J. Wang, and J. Shang, "Strategies for integral metabolism profile of multiple compounds in herbal medicines: pharmacokinetics, metabolites characterization and metabolic interactions," Current Drug Metabolism, vol. 12, no. 9, pp. 809-817, 2011.

[70] C. Y. Li, L. W. Qi, and P. Li, "Correlative analysis of metabolite profiling of Danggui Buxue Tang in rat biological fluids by rapid resolution LC-TOF/MS," Journal of Pharmaceutical and Biomedical Analysis, vol. 55, no. 1, pp. 146-160, 2011.

[71] C. Xiang, X. Qiao, Q. Wang, D. Guo, and M. Ye, "From single compounds to herbal extract: a strategy to systematically characterize the metabolites of licorice in rats," Drug Metabolism and Disposition, vol. 39, no. 9, pp. 1597-1608, 2011.

[72] X. Qiao, M. Ye, C. Xiang, Q. Wang, W.-J. Miao, and D.A. Guo, "Analytical strategy to reveal the in vivo process of multi-component herbal medicine: a pharmacokinetic study of licorice using liquid chromatography coupled with triple quadrupole mass spectrometry," Journal of Chromatography A, vol. 1258, pp. 84-93, 2012.

[73] P. Gong, N. Cui, L. Wu et al., "Chemicalome and metabolome matching approach to elucidating biological metabolic networks of complex mixtures," Analytical Chemistry, vol. 84, no. 6, pp. 2995-3002, 2012.

[74] A. K. Smilde, J. A. Westerhuis, H. C. J. Hoefsloot et al., "Dynamic metabolomic data analysis: a tutorial review," Metabolomics, vol. 6, no. 1, pp. 3-17, 2010.

[75] J. A. Westerhuis, E. J. J. van Velzen, H. C. J. Hoefsloot, and A. K. Smilde, "Multivariate paired data analysis: multilevel PLSDA versus OPLSDA," Metabolomics, vol. 6, no. 1, pp. 119-128, 2010.

[76] J. van Duynhoven, E. E. Vaughan, D. M. Jacob et al., "Metabolic fate of polyphenols in the human superorganism," Proceedings of the National Academy of Sciences of the United States of America, vol. 108, supplement 1, pp. 4531-4538, 2011.

[77] J. P. M. van Duynhoven, E. J. J. van Velzena, and J. A. Westerhuis, "Nutrikinetics: concept, technologies, applications, perspectives," Trends in Food Science and Technology, vol. 26, no. 1, pp. 4-13, 2012.

[78] R. Llorach, M. Garcia-Aloy, S. Tulipani, and C. Andres-Lacueva, "Nutrimetabolomic strategies to develop new biomarkers of intake and health effects," Journal of Agricultural and Food Chemistry, vol. 60, no. 36, pp. 8797-8808, 2012.

[79] E. M. Lenz and I. D. Wilson, "Analytical strategies in metabonomics," Journal of Proteome Research, vol. 6, no. 2, pp. 443-458, 2007.

[80] J. van der Greef, "Perspective: all systems go," Nature, vol. 480, no. 7378, p. S87, 2011.

[81] T. Ideker, T. Galitski, and L. Hood, "A new approach to decoding life: systems biology," Annual Review of Genomics and Human Genetics, vol. 2, pp. 343-372, 2001.
[82] H. Kitano, “Systems biology: a brief overview," Science, vol. 295, no. 5560, pp. 1662-1664, 2002.

[83] K. Lan, Y. Zhang, J. Yang, and L. Xu, "Simple quality assessment approach for herbal extracts using high performance liquid chromatography-UV based metabolomics platform," Journal of Chromatography A, vol. 1217, no. 8, pp. 1414-1418, 2010.

[84] Z. Wang, H. Hu, F. Chen et al., "Metabolic profiling assisted quality assessment of Rhodiola rosea extracts by highperformance liquid chromatography," Planta Medica, vol. 78, no. 7, pp. 740-746, 2012.

[85] L.-X. Duan, M. Li, M. Chen et al., "Use of the metabolomics approach to characterize chinese medicinal material Huangqi," Molecular Plant, vol. 5, no. 2, pp. 376-386, 2012.

[86] M. Wang, R. J. A. N. Lamers, H. A. A. J. Korthout et al., "Metabolomics in the context of systems biology: Bridging Traditional Chinese Medicine and molecular pharmacology," Phytotherapy Research, vol. 19, no. 3, pp. 173-182, 2005.

[87] A. Zhang, H. Sun, Z. Wang, W. Sun, P. Wang, and X. Wang, "Metabolomics: towards understanding traditional Chinese medicine," Planta Medica, vol. 76, no. 17, pp. 2026-2035, 2010.

[88] X. Wang, H. Sun, A. Zhang, W. Sun, P. Wang, and Z. Wang, "Potential role of metabolomics apporoaches in the area of traditional Chinese medicine: as pillars of the bridge between Chinese and Western medicine," Journal of Pharmaceutical and Biomedical Analysis, vol. 55, no. 5, pp. 859-868, 2011.

[89] P. Liu, S. Liu, D. Tian, and P. Wang, "The applications and obstacles of metabonomics in traditional Chinese medicine," Evidence-Based Complementary and Alternative Medicine, vol. 2012, Article ID 945824, 4 pages, 2012.

[90] T. Wu, M. Yang, H.-F. Wei, S.-H. He, S.-C. Wang, and G. Ji, "Application of metabolomics in traditional chinese medicine differentiation of deficiency and excess syndromes in patients with diabetes mellitus," Evidence-Based Complementary and Alternative Medicine, vol. 2012, Article ID 968083, 11 pages, 2012.

[91] M. Jové, J. C. E. Serrano, N. Ortega et al., "Multicompartmental LC-Q-TOF-based metabonomics as an exploratory tool to identify novel pathways affected by polyphenol-rich diets in mice," Journal of Proteome Research, vol. 10, no. 8, pp. 3501-3512, 2011.

[92] P. J. Turnbaugh, R. E. Ley, M. Hamady, C. M. Fraser-Liggett, R. Knight, and J. I. Gordon, "The Human Microbiome Project," Nature, vol. 449, no. 7164, pp. 804-810, 2007.

[93] J. Qin, R. Li, J. Raes et al., "A human gut microbial gene catalogue established by metagenomic sequencing," Nature, vol. 464, no. 7285, pp. 59-65, 2010.

[94] M. Arumugam, J. Raes, E. Pelletier et al., "Enterotypes of the human gut microbiome," Nature, vol. 473, no. 7346, pp. 174-180, 2011.

[95] T. H. M. P. Consortium, "A framework for human microbiome research," Nature, vol. 486, no. 7402, pp. 215-221, 2012.

[96] T. H. M. P. Consortium, "Structure, function and diversity of the healthy human microbiome," Nature, vol. 486, no. 7402, pp. 207-214, 2012.

[97] I. Rosabelle McManus, A. O. Contag, and R. E. Olson, "Characterization of endogenous ethanol in the mammal," Science, vol. 131, no. 3393, pp. 102-103, 1960.

[98] G. Spinucci, M. Guidetti, E. Lanzoni, and L. Pironi, "Endogenous ethanol production in a patient with chronic intestinal pseudo-obstruction and small intestinal bacterial overgrowth," European Journal of Gastroenterology and Hepatology, vol. 18, no. 7, pp. 799-802, 2006. 
[99] D. Compare, P. Coccoli, A. Rocco, O. M. Nardone, S. De Maria, and G. Nardone, "Gut-liver axis: the impact of gut microbiota on non alcoholic fatty liver disease," Nutrition, Metabolism and Cardiovascular Diseases, vol. 22, no. 6, pp. 471-476, 2012.

[100] F. P. J. Martin, M. E. Dumas, Y. Wang et al., "A top-down systems biology view of microbiome-mammalian metabolic interactions in a mouse model," Molecular Systems Biology, vol. 3, article 112, 2007.

[101] F. P. J. Martin, Y. Wang, N. Sprenger et al., "Probiotic modulation of symbiotic gut microbial-host metabolic interactions in a humanized microbiome mouse model," Molecular Systems Biology, vol. 4, article 157, 2008.

[102] F. P. J. Martin, N. Sprenger, I. K. S. Yap et al., "Panorganismal gut microbiome-host metabolic crosstalk," Journal of Proteome Research, vol. 8, no. 4, pp. 2090-2105, 2009.

[103] F. P. J. Martin, Y. Wang, I. K. S. Yap et al., “Topographical variation in murine intestinal metabolic profiles in relation to microbiome speciation and functional ecological activity," Journal of Proteome Research, vol. 8, no. 7, pp. 3464-3474, 2009.

[104] X. Zheng, G. Xie, A. Zhao et al., "The footprints of gut microbial-mammalian co-metabolism," Journal of Proteome Research, vol. 10, no. 12, pp. 5512-5522, 2011.

[105] J. K. Nicholson, E. Holmes, R. Burcelin, and S. Pettersson, "Host-gut microbiota metabolic interactions," Science, vol. 336, no. 6086, pp. 1262-1267, 2012.

[106] I. D. Wilson and J. K. Nicholson, "The role of gut microbiota in drug response," Current Pharmaceutical Design, vol. 15, no. 13, pp. 1519-1523, 2009.

[107] T. A. Clayton, D. Baker, J. C. Lindon, J. R. Everett, and J. K. Nicholson, "Pharmacometabonomic identification of a significant host-microbiome metabolic interaction affecting human drug metabolism," Proceedings of the National Academy of Sciences of the United States of America, vol. 106, no. 34, pp. 14728-14733, 2009.

[108] W. Meinl, S. Sczesny, R. Brigelius-Flohé, M. Blaut, and H. Glatt, "Impact of gut microbiota on intestinal and hepatic levels of phase 2 xenobiotic-metabolizing enzymes in the rat," Drug Metabolism and Disposition, vol. 37, no. 6, pp. 1179-1186, 2009.

[109] B. Björkholm, C. M. Bok, A. Lundin, J. Rafter, M. L. Hibberd, and S. Pettersson, "Intestinal microbiota regulate xenobiotic metabolism in the liver," PLoS ONE, vol. 4, no. 9, Article ID e6958, 2009.

[110] S. Peters, H. G. Janssen, and G. Vivó-Truyols, “Trend analysis of time-series data: a novel method for untargeted metabolite discovery," Analytica Chimica Acta, vol. 663, no. 1, pp. 98-104, 2010 . 


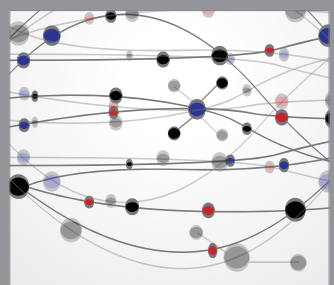

The Scientific World Journal
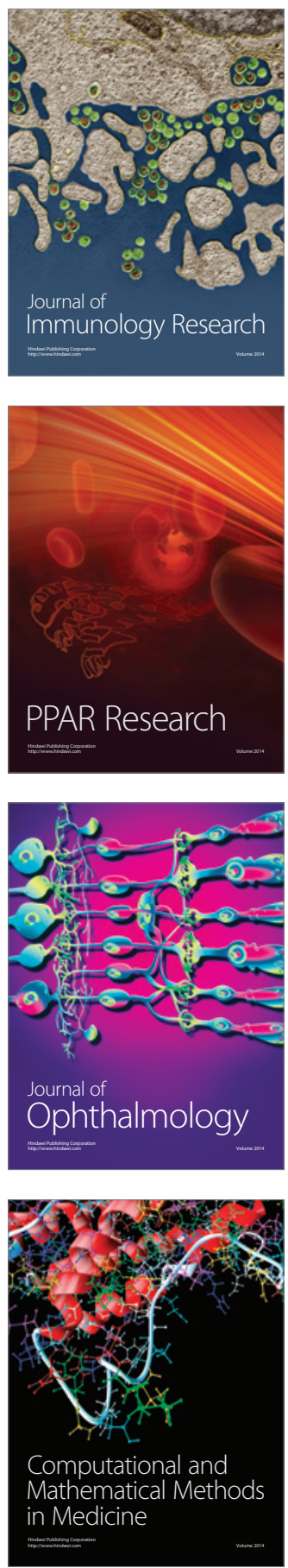

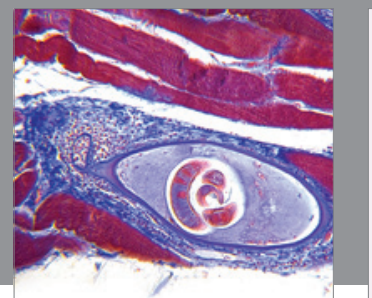

Gastroenterology

Research and Practice
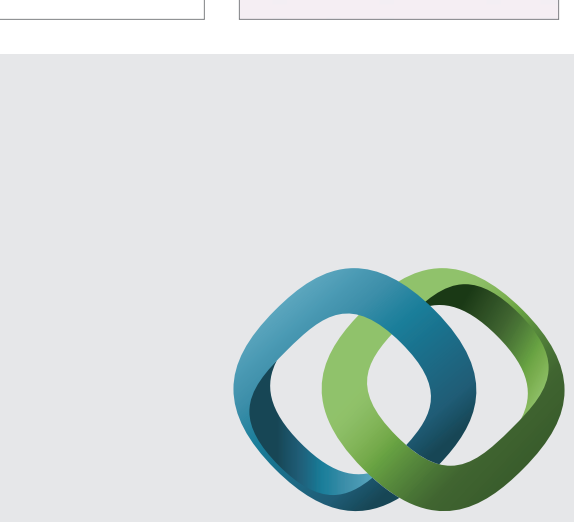

\section{Hindawi}

Submit your manuscripts at

http://www.hindawi.com
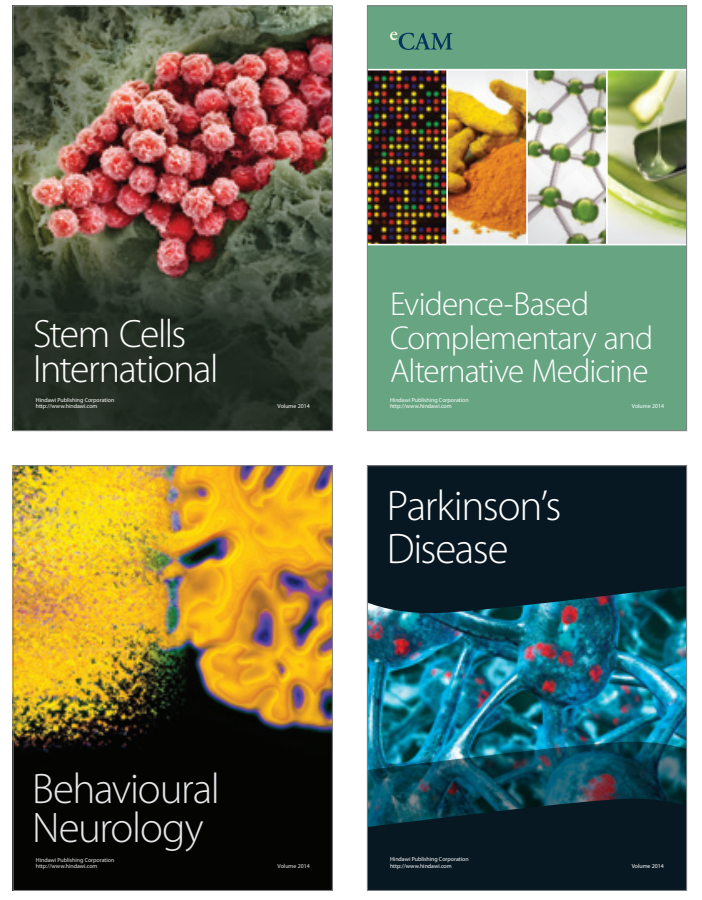
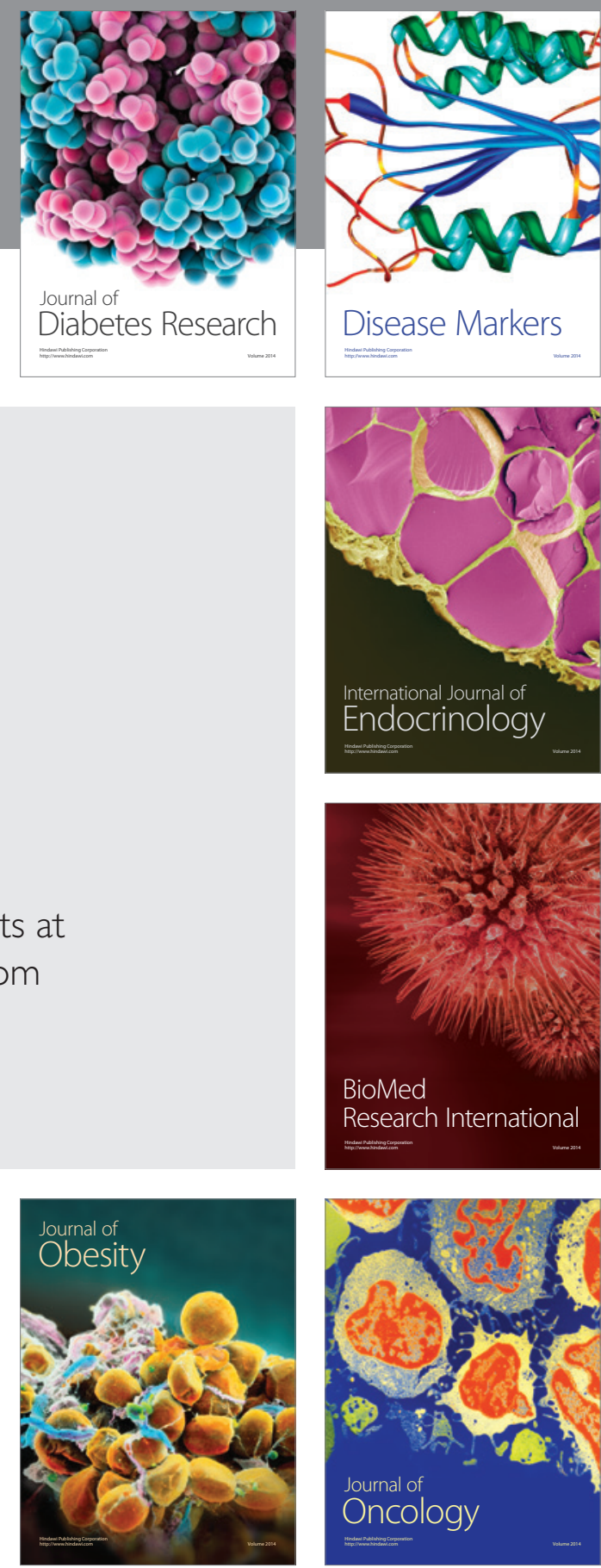

Disease Markers
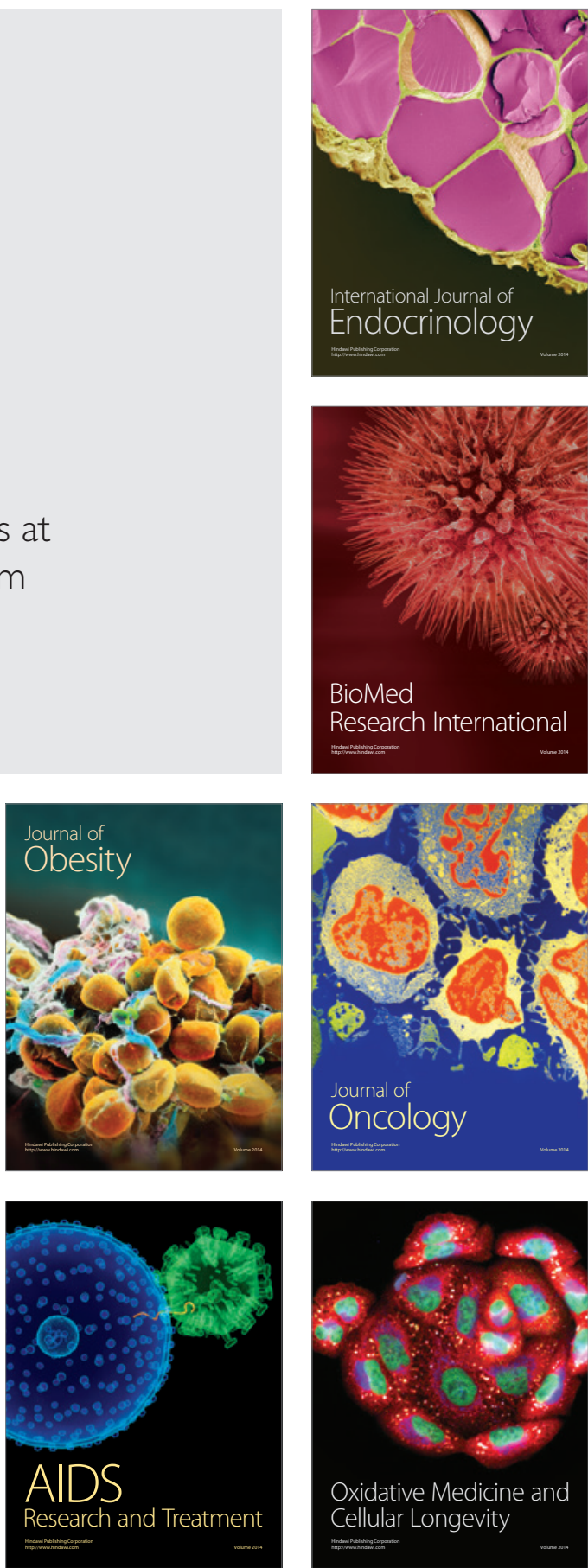